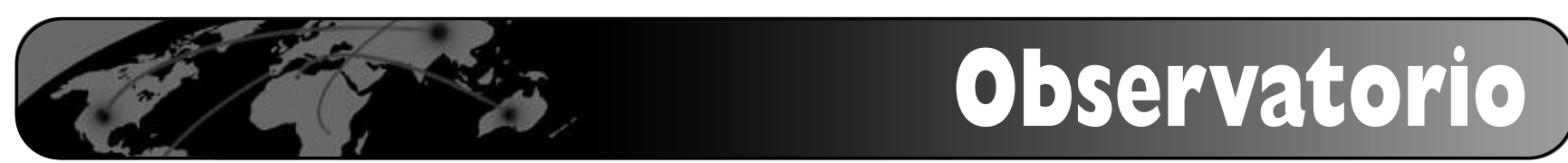

\title{
¿Intranets? Decididamente sí, pero...
}

\author{
Por Cristina Soy Aumatell
}

LAS INTRANETS O PORTALES CORPORATIVOS, gozan ya de una cierta trayectoria en las organizaciones grandes y medianas ${ }^{1}$, públicas ${ }^{2}$ y privadas, industriales y de servicios, $y$ en esta nota queremos plantear algunas reflexiones destinadas a analizar si están contribuyendo a la mejora de la gestión de la información en las entidades donde se hallan implantadas.

Según se desprende del estudio Las TIC como agente de cambio en la empresa española. Situación actual y tendencias de futuro ${ }^{3}$ que analiza las inversiones en tecnologías de la información de los últimos tres años, las partidas presupuestarias destinadas a intranets y extranets han aumentado en el $70 \%$ de las empresas, no han experimentado cambios en el $24 \%$ y en el $6 \%$ han disminuido.

Los datos actuales disponibles permiten a priori, a la luz de la tendencia de las inversiones en la empresa, constatar un interés en la herramienta, la progresiva implantación en el segmento pyme (aunque se halla aún lejos del acceso universal a todos los empleados), y la difusión e integración de servicios en la administración local, la más numerosa y próxima al ciudadano.

\section{Diferente percepción de una intranet}

En primer lugar queremos señalar que existen tantos visiones o modelos de intranets como organizaciones, si entendemos éstas como entornos únicos integrados por unas personas, una cultura corporativa, unos procesos y objetivos, unas tecnologías, con un tamaño, y unos recursos determinados, entre otros factores. Las organizaciones, con sus matices y evoluciones, han planteado las intranets como espacios digitales de comunicación, contribución y acceso a información, de lo que resulta principalmente un sistema de organización y difusión de la información, inicialmente con contenido puro, y progresivamente o paralelamente con servicios asociados elevando notablemente su grado de sofisticación y prestaciones. A menudo, sin embargo, el principal objetivo de la intranet corporativa se asocia a la difusión eficiente y oportuna de información a toda la organización, un objetivo focalizado en una actividad, no en un resultado, y por tanto alejado y no alineado con la dirección estratégica de la institución.
Este planteamiento dificulta que la intranet pueda posicionarse como un activo estratégico que responda a las necesidades a corto y largo plazo de la organización. En este sentido, el desarrollo de una intranet no debe obviar el substrato teórico de modelos de amplia tradición en la gestión de la información basados en la importancia del entorno de la información y el comportamiento informacional de los usuarios. Nos referimos a la ecología de la información ${ }^{4}$, los avances en necesidades y usos de la información propuestos por Taylor $^{5}$ y los procesos de valor añadido.

La ecología de la información es el entorno informativo de la organización, integrado por numerosos subsistemas sociales, culturales y políticos que interactúan y son interdependientes dando forma a la creación, flujo y uso de la información en la organización. La ecología influye en la forma en que la información es producida y almacenada, en su disponibilidad y destinatario, en la requerida y valorada en el marco del desempeño.

\section{Intranet modelada por la ecología informativa}

En el diseño de una intranet los elementos que deben analizarse son:

- papel de la información y la contribución de sus recursos y servicios a la misión corporativa;

—objetivos de la intranet y como contribuirá a la misión de la organización;

— planes, políticas, procedimientos de gestión de la información;

—cultura informativa;

- estructuras organizativas y su influencia en la gestión y en la forma de compartir información; y

—organización física, el personal y las normas y rutinas establecidas para la gestión de la información y el mantenimiento de la memoria corporativa.

Desde la subdisciplina del análisis de necesidades y usos de la información en primer lugar se obtiene una aproximación lo más exacta posible de quiénes son los usuarios en términos de necesidades y características de la solicitud de información; en segundo lugar hay 
que entender la estructura de la situaciones laborales problemáticas a las que se enfrentan los usuarios; $y$, por último se examinan los comportamientos y prácticas que éstos utilizan para resolver dichas situaciones.

Partiendo de estas aproximaciones las intranets deben se concebidas como procesos de valor añadido que amplían y extienden el valor de la información a la organización y a sus usuarios. ¿Cómo? A nivel de tareas, la intranet debe ser diseñada para dar soporte a los comportamientos informacionales de los usuarios y resolver las situaciones problemáticas profesionales que se les puedan plantear. A nivel organizativo, las aplicaciones y los servicios deben ser concebidos para alcanzar o mejorar la ecología de la información a la que nos referíamos antes. La intranet proporciona un espacio único en el que los usuarios acceden a contenidos, se comunican y colaboran con terceros. Su valor también radica en las posibilidades para compartir, convertir y utilizar de forma combinada conocimiento explícito, tácito y corporativo. Los procesos de toma de decisión se benefician de la estructuración de los procesos en base a rutinas y directrices.

\section{Utilidad y usabilidad}

La consultora DiamondCluster establecía: "El valor real de un portal es su habilidad para crear un ambiente eficiente que permita a los usuarios colaborar, realizar tareas y obtener información de una sola fuente personalizable", y definía como sus principales atributos:

—buscabilidad;

— categorización, estructura y organización de la información;

—editabilidad concebida como un sistema simple de generación y publicación digital de los contenidos;

-integración o transparencia entre las bases de datos internas y externas;

— personalización y adecuación de acuerdo con perfiles establecidos;

- colaboración o interacción entre los empleados;

—seguridad que establece quién puede acceder a qué.

Sin duda las intranets han hecho omnipresentes términos como contenido $^{6}$, han sido motores del desarrollo de ámbitos como la gestión de contenidos, la arquitectura de la información, la usabilidad, la accesibilidad, etc., y han creado o refundado nuevos modelos de negocio como la sindicación de contenidos. De la mano de la gestión de contenidos muchas organizaciones han conocido las ventajas de la nada nueva gestión documental cuando se les ha planteado la necesidad de gestionar de forma integrada la información producida en la organización, que puede ser incorporada, o no, a la intranet en algún estadio, coordinarse con la Web pública, e implementar una eventual extranet para clientes o proveedores o para cualquier otro grupo de interés.

\section{No es fácil implantar una intranet con éxito}

A pesar del camino recorrido y del corpus teórico y de práctica profesional del que se dispone, las cifras sobre el nivel de uso de las intranets que a menudo se han divulgado no son nada alentadoras. La firma de investigación y asesoramiento americana de la industria de la información Outsell, señalaba que sólo entre un 15-20\% de los empleados leen el contenido de la intranet, cuando se considera según otras fuentes que el éxito se alcanza cuando el $40 \%$ de los usuarios potenciales la utilizan a diario.

Algunas de las intranets más utilizadas son aquellas que inciden en gestiones u operativas que el usuario considera relevantes y que están muy vinculadas con la organización y para las cuales se habilita como único canal. Es el caso de los portales del empleado, que combinan básicamente comunicación interna con trámites y gestiones con el propósito de "hacer la vida más fácil a la plantilla" . Los portales del empleado contribuyen visiblemente a la mejora de la productividad y de la administración de personal, incorporando: —gestión de vacaciones y permisos;

—nómina virtual;

— control de presencias;

-inscripción en cursos;

— cambios en la situación familiar;

- preguntas sobre beneficios;

—comunicaciones de los empleados.

En términos de mejora de la administración incluyen: —control y análisis del presupuesto;

- gestión de compras;

—gestión de gastos;

—gestión de viajes;

—aprobación de permisos;

—modificación de actividades de los empleados;

—obtención de informes de gestión.

Una de las claves del escaso uso de las intranets nos la aporta la ley de Mooers: una persona no utiliza un sistema de información si hacerlo no le aporta un beneficio, o no es socialmente recompensado en el entorno concreto (con el reconocimiento del colectivo).

Ello nos lleva a referirnos a la cultura de la información de la organización y al hecho que la institución considere crítico disponer y usar información, se incentive la creación de entornos informativamente in- 
tensivos, en los que se asimile, se comparta y se difunda correctamente como una de las claves interpretativas del uso que se da a las intranets en determinadas organizaciones y entornos.

Adicionalmente, la asignatura pendiente de muchas intranets continúa siendo incentivar la contribución, lo que al margen de lo comentado anteriormente, requiere establecer modelos de retribución claros y tangibles junto a una definición de procesos y a la tecnología facilitadora adecuada.

\section{Mejora de la comunicación}

Las intranets por otro lado, están contribuyendo a una gestión más integrada y global de la información interna, externa y, en menor grado, corporativa de las organizaciones, y a un mayor grado de procedimentación, a la disponibilidad de información seleccionada, filtrada y contextualizada, de alta calidad que incorpora la oferta de proveedores tradicionales de información científica, tecnológica y empresarial y nuevos entrantes.

Asimismo, están facilitando la comunicación interna, la divulgación de las prácticas y procedimientos, la formación virtual y también presencial o el trabajo colaborativo, lo que permite mejorar determinados flujos y procesos de trabajo.

Están simplificando enormemente el proceso de publicación de información dotando a los usuarios de medios de creación y de compartir información y conocimiento. La facilidad de edición de contenidos se ha visto acentuada con herramientas como los blogs y los wikis, aún poco extendidos en las organizaciones españolas a nivel interno y que ilustran la sencillez para publicar y compartir contenidos digitales en la red. El profesional de la información se ha convertido en un agente dinamizador de la intranet corporativa y motor de la mejora continua de la misma, editor y creador de nuevos contenidos, organizador y analista de contenidos, que difunde el potencial de la intranet buscando visibilidad a su labor.

Si bien han supuesto la descentralización parcial a nivel operativo de la gestión de la información principalmente interna, el autor del documento se convierte en editor del mismo y usuario, y se han extendido entre el colectivo de usuarios términos técnicos tales como catalogación, indexación, taxonomías o clasificación. La gestión de la información y de contenidos no se considera generalmente una competencia transversal y sus tareas no se asumen asociadas al puesto de trabajo concreto, sino aún como una carga de trabajo adicional.

Las intranets, integradas en las rutinas de trabajo diario, nos brindan la oportunidad de integrar la ges- tión personal de la información en las políticas y estrategias globales de gestión de la información de las organizaciones. Esto, desde la óptica meramente informativa, otorga a las intranets un alcance e impacto mayor que el de la simple publicación de contenidos y consumo de información, incrementando así el nivel de cultura de la información y alfabetismo informacional de los usuarios y la valoración de la función información en las organizaciones.

\section{Bibliografía}

Alòs-Móner, Adela d'. "Intranets: sonrisas y lágrimas". En: El profesional de la información, 2002, enero-febrero, v. 11, n. 1, pp. 4-8.

Choo, Chun Wei; Detlor, Brian; Turnbull, Don. Web work: information seeking and knowledge work on the World Wide Web. Dordrecht: Kluwer, 2000. ISBN 0-7923-6460-0

Tredinnick, Like. Why intranets fail (and how to fix them): a practical guide for information professionals. Oxford: Chandos Publishing, 2004. ISBN 1-84334-068-2

\section{Notas}

1. La Escuela de Organización Industrial (EOI) ha analizado el grado de desarrollo e implantación de las TIC (tecnologías de la información y la comunicación) en las pequeñas y medianas empresas españolas, el $96 \%$ del tejido empresarial, a partir de una muestra formada por 751 empresas de más de 19 empleados. Del estudio del año 2005 se desprende el grado de acceso que la pyme española proporciona a la intranet corporativa. Se indica el porcentaje de empresas y el correspondiente porcentaje de empleados con acceso

$17,2 \%$ entre el $76 \%$ y el $100 \%$

$6,6 \%$ entre el $51 \%$ y el $75 \%$

$12,0 \%$ entre el $26 \%$ y el $50 \%$

$20,9 \%$ entre el $10 \%$ y el $25 \%$

$17,5 \%$ menos del $10 \%$

$23,9 \%$ no da acceso a ninguno de sus empleados

2. En el caso de los ayuntamientos españoles las aplicaciones informáticas accesibles desde la intranet son principalmente las relacionadas con la gestión interna como son la gestión del personal y alcaldía en el caso de los ayuntamientos de capitales de provincia y las que implican información o relaciones con el exterior en los municipios no capitales de provincia tales como padrón y censo, legislación, cartografía digital. Fuente: eEspaña 2006: informe anual sobre el desarrollo de la sociedad de la información en España. Madrid: Fundación France Telecom.

3. Valor, Josep; Sieber, Sandra. Las TIC como agente de cambio en la empresa española. Situación actual y tendencias de futuro. Barcelona: IESE; PriceWaterhouseCoopers (Cuadernos del Ebcenter).

4. Davenport, Thomas. Information ecology: mastering the information and knowledge environment. New York: Oxford University Press, 1997. ISBN 0-19-511168-0

5. Taylor, Robert. "Information use environments". En: Progress in communication sciences, 1991, v. 10, pp. 217-255.

MacMullin, S.; Taylor, Robert. «Problem dimensions and information traits». En: Information society, 1984, v. 3, n. 1, pp. 91-111.

6. Con la popularización hace unos años de frases como: "Content is King and Context is Queen"

7. Simón, Cristina; Cabanas, Custodia. "Desarrollo actual y perspectivas del portal del empleado en las empresas españolas (II), Capital humano, 2004, n. 181, octubre, pp. 80-90.

8. Calvin Mooers

http://en.wikipedia.org/wiki/Calvin_Mooers

Cristina Soy i Aumatell, Caixa Holding, SA. csoy@caixaholding.es 